T. Urata

Nagoya Math. J.

Vol. 81 (1981), 91-103

\title{
HOLOMORPHIC AUTOMORPHISMS AND CANCELLATION THEOREMS
}

\author{
TOSHIO URATA
}

\section{§1. Statement of the result}

In this note, complex analytic spaces are always assumed to be reduced and connected.

Let $X$ be a complex analytic space of positive dimension and $A$ a complex analytic subvariety of $X$. We call $A$ a direct factor of $X$ if there exist a complex analytic space $B$ and a biholomorphic mapping $f: A \times B$ $\rightarrow X$ such that, for some $b \in B, f(a, b)=a$ on $A$, and a complex analytic space $X$ to be primary if $X$ has no direct factor, not equal to $X$ itself, of positive dimension. By a primary decomposition of $X$, we mean a cartesian product $X_{1} \times X_{2} \times \cdots \times X_{n}$ of primary complex analytic spaces $X_{1}, X_{2}, \cdots$, $X_{n}$ of positive dimension, such that $X_{1} \times X_{2} \times \cdots \times X_{n}$ is biholomorphic to $X$. We shall give examples of primary decomposition in $\S 7$.

Now, consider the following condition (C) for an arbitrary complex analytic space $X$ :

Given arbitrarily a complex analytic space $Y$ and a holomorphic

(C) mapping $\phi: Y \times X \rightarrow X$, if $\phi\left(y_{0}, \cdot\right): X \rightarrow X$ is a biholomorphic mapping for some $y_{0} \in Y$ then

$$
\phi(y, \cdot)=\phi\left(y_{0}, \cdot\right) \quad \text { on } X \text { for every } y \in Y .
$$

We denote by $C$ the collection of all complex analytic spaces which satisfy the condition $(\mathrm{C})$.

Throughout this note we are concerned with two classes of complex analytic spaces, (1) hyperbolic complex analytic spaces in the sense of Kobayashi [4] and (2) compact complex analytic spaces of general type (see the following, extracted from Ueno [10]).

Let $M$ be a compact complex analytic manifold with the canonical line bundle $K(M)$. The Kodaira dimension $\kappa(M)$ of $M$ is equal to an in-

Received June 18, 1979. 
teger $\kappa\left(0 \leqslant \kappa \leqslant \operatorname{dim}_{C} M\right)$ provided that

$$
0<\limsup _{m \rightarrow+\infty} \frac{\operatorname{dim}_{C} H^{0}(M, m K(M))}{m^{x}}<\infty,
$$

or $-\infty$ otherwise. Note that the Kodaira dimension is a bimeromorphic invariant of compact complex analytic manifolds. For a compact irreducible complex analytic space $X$, the Kodaira dimension $k(X)$ of $X$ is defined as the Kodaira dimension of any desingularization of $X$. A compact complex analytic space $X$ is called of general type (or of hyperbolic type in the terminology of [10]), if $X$ is irreducible and $\kappa(X)=\operatorname{dim}_{C} X$.

We shall show the following in $\S 2$.

Proposition 1 (cf. Royden [9]). Every hyperbolic complex analytic space is contained in $C$.

Proposition 2. Every compact complex analytic space of general type is contained in $C$.

Proposition 3. Let $X$ and $Y$ be complex analytic spaces. Then the product $X \times Y$ is contained in $C$ if and only if $X, Y$ are contained in $C$.

And, we shall prove in $\S 4$

THEOREM 1. Take an arbitrary complex analytic space $X$ of positive dimension, which is a member of $C$. Then $X$ possesses a unique primary decomposition $X_{1} \times \cdots \times X_{n}$. Here, the uniqueness means that if $Y_{1} \times \cdots$ $\times Y_{m}$ is another primary decomposition of $X$ then (1) $m=n$ and (2) for any biholomorphic mapping $f$ of $X_{1} \times \cdots \times X_{n}$ to $Y_{1} \times \cdots \times Y_{n}$ there are biholomorphic mappings $f_{i}: X_{i} \rightarrow Y_{i}(i=1, \cdots, n)$ such that $f=f_{1} \times \cdots \times$ $f_{n}$ on $X_{1} \times \cdots \times X_{n}$ after a suitable reordering of $Y_{1}, \cdots, Y_{n}$.

Corollary 1. Let $X, Y$ and $V$ be complex analytic spaces of the collection $C$. If $V \times X$ is biholomorphic to $V \times Y$, then $X$ is biholomorphic to $Y$.

For an arbitrary complex analytic space $X$, let Aut $(X)$ denote the group of all biholomorphic mappings of $X$ to $X$ itself.

Let $X$ be a complex analytic space of the collection $C$ and assume that

$$
X=\underbrace{X_{1} \times \cdots \times X_{1}}_{n_{1} \text { terms }} \times \underbrace{X_{2} \times \cdots \times X_{2}}_{n_{2} \text { terms }} \times \cdots \times \underbrace{X_{p} \times \cdots \times X_{p}}_{n_{p} \text { terms }}
$$


$\left(n_{1}+n_{2}+\cdots+n_{p}=n\right)$ for primary complex analytic spaces $X_{1}, X_{2}, \cdots, X_{p}$ which are not biholomorphic to each other. Consider the subgroup $G$ of Aut $(X)$ which is induced naturally by permutations, namely, $\sigma \in G$ if and only if

$$
\sigma\left(x_{1}, x_{2}, \cdots, x_{n}\right)=\left(x_{\sigma_{1}}, x_{\sigma_{2}}, \cdots, x_{\sigma_{n}}\right) \quad \text { on } X
$$

for some permutation $\left(\sigma_{1}, \sigma_{2}, \cdots, \sigma_{n}\right)$ of $(1,2, \cdots, n)$ such that

$$
\left\{\sigma_{n_{1}+\cdots+n_{i-1}+1}, \cdots, \sigma_{n_{1}+\cdots+n_{i}}\right\}=\left\{n_{1}+\cdots+n_{i-1}+1, \cdots, n_{1}+\cdots+n_{i}\right\}
$$

Obviously $G$ is group-isomorphic to a direct product of some symmetric groups. Immediately from Theorem 1, we obtain

Corollary 2. For each $f \in \operatorname{Aut}(X)$, there exist uniquely $f_{n_{1}+\cdots+n_{i-1}+1}$, $\cdots, f_{n_{1}+\cdots+n_{i}} \in \operatorname{Aut}\left(X_{i}\right)(i=1, \cdots, p)$ and $\sigma \in G$ such that

$$
f=\sigma \circ\left(f_{1} \times \cdots \times f_{n}\right) \quad \text { on } X \text {. }
$$

This means that Aut $(X)$ is a semidirect product of $G$ and a normal subgroup

$$
\begin{aligned}
\underbrace{\operatorname{Aut}\left(X_{1}\right) \times \cdots \times \operatorname{Aut}\left(X_{1}\right)}_{n_{1} \text { terms }} & \times \underbrace{\operatorname{Aut}\left(X_{2}\right) \times \cdots \times \operatorname{Aut}\left(X_{2}\right)}_{n_{2} \text { terms }} \\
& \times \cdots \times \underbrace{\operatorname{Aut}\left(X_{p}\right) \times \cdots \times \operatorname{Aut}\left(X_{p}\right)}_{n_{p} \text { terms }}
\end{aligned}
$$

of $\operatorname{Aut}(X)$.

Note that the above Corollary 2 implies Satz 3.4 of Peters [8], which is a generalization of a theorem of $\mathrm{H}$. Cartan. We shall prove the following cancellation theorems in $\S 5$ and $\S 6$.

Theorem 2. Let $X, Y$ and $V$ be complex analytic spaces such that $V \times X$ is biholomorphic to $V \times Y$. If $V$ is hyperbolic, then $X$ is biholomorphic to $Y$.

Theorem 3. Let $V$ be a compact complex analytic space of general type. Suppose that $X$ and $Y$ are compact irreducible complex analytic spaces such that $V \times X$ is biholomorphic to $V \times Y$. Then $X$ is biholomorphic to $Y$.

The author would like to express his sincere thanks to Professor H. Fujimoto for his valuable suggestions. 


\section{§2. Proofs of Propositions}

Proof of Proposition 1. Let $X$ be a hyperbolic complex analytic space. Take arbitrarily a complex analytic space $Y$ and a holomorphic mapping $\phi: Y \times X \rightarrow X$ such that $\phi\left(y_{0}, \cdot\right) \in \operatorname{Aut}(X)$ for some $y_{0} \in Y$. We have to prove that $\phi(y, \cdot)=\phi\left(y_{0}, \cdot\right)$ on $X$ for every $y \in Y$. Since $Y$ is connected, every two points of $Y$ can be connected by a chain of holomorphic mappings of the unit open disc $D$ (in the complex line $C$ ) into $Y$ (cf. Kobayashi $[4]$, p. 97). Hence it suffices to prove the above for the case $Y=D$. Suppose that $Y=D$. Without loss of generality, we may assume that $\phi\left(z_{0}, \cdot\right)$ $=$ identity on $X$ for some $z_{0} \in D$. Let $S$ be the set of all singular points of $X$ and take an arbitrary point $x_{0}$ of $X-S$. Define the holomorphic mappings $\phi_{n}(n=1,2, \cdots)$ of $D$ into $X$ as follows:

$$
\phi_{1}(z)=\phi\left(z, x_{0}\right) \quad \text { and } \quad \phi_{n+1}(z)=\phi\left(z, \phi_{n}(z)\right) \quad \text { on } D(n=1,2, \cdots) .
$$

Obviously, $\phi_{n}\left(z_{0}\right)=x_{0}$ for $n=1,2, \cdots$. Now, fix holomorphic local coordinates at $x_{0}$ in $X$. Since $X$ is hyperbolic, the family $\left\{\phi_{n}\right\}_{n>0}$ is equicontinuous on $D$. This implies that, for every positive integer $\ell$, there exists a positive constant $A_{\ell}$ such that

$$
\left\|\frac{d^{\ell} \phi_{n}}{d z^{\ell}}\left(z_{0}\right)\right\| \leqslant A_{\ell} \quad(n=1,2, \cdots) .
$$

On the other hand, since $\phi\left(z_{0}, x\right)=x$ on $X$, the mapping $\phi$ has a power series expansion of the form

$$
\phi(z, x)=x+a(x)\left(z-z_{0}\right)^{\ell}+O\left(\left(z-z_{0}\right)^{\ell+1}\right)
$$

near the point $\left(z_{0}, x_{0}\right)$ in $D \times X$. Then $\phi_{n}$ has the expansion

$$
\phi_{n}(z)=x_{0}+n a\left(x_{0}\right)\left(z-z_{0}\right)^{\ell}+O\left(\left(z-z_{0}\right)^{\ell+1}\right)
$$

near $z_{0}$ in $D(n=1,2, \cdots)$. Thus we see that

$$
\ell ! n\left\|a\left(x_{0}\right)\right\|=\left\|\frac{d^{\ell} \phi_{n}}{d z^{\ell}}\left(z_{0}\right)\right\| \leqslant A_{\ell} \quad(n=1,2, \cdots) .
$$

Hence $a\left(x_{0}\right)=0$. This means that $\phi(z, x)=x$ locally at $\left(z_{0}, x_{0}\right)$ in $D \times X$. Since $D$ is connected and $S$ is nowhere dense in $X$, we see that $\phi(z, x)=$ $x$ on $D \times X$. This completes the proof.

Proof of Proposition 2. Let $X$ be a compact complex analytic space of general type. Take arbitrarily a complex analytic space $Y$ and a holo- 
morphic mapping $\phi: Y \times X \rightarrow X$ such that $\phi\left(y_{0}, \cdot\right) \in$ Aut $(X)$ for some $y_{0} \in Y$. We have to prove that $\phi(y, \cdot)=\phi\left(y_{0}, \cdot\right)$ on $X$ for every $y \in Y$. Let $\mathrm{Hol}(X, X)$ be the set of all holomorphic mappings of $X$ into $X$ and consider the mapping

$$
\tilde{\phi}: Y \rightarrow \operatorname{Hol}(X, X)
$$

defined by the formula $\tilde{\phi}(y)=\phi(y, \cdot) \in \operatorname{Hol}(X, X)$ for each $y \in Y$. Obviously, $\tilde{\phi}$ is a continuous mapping into the space $\operatorname{Hol}(X, X)$ equipped with the compact-open topology. It is well known that $\operatorname{Aut}(X)$ is open in $\operatorname{Hol}(X, X)$. On the other hand, by Corollary 14.3 of Ueno [10], Aut $(X)$ is finite. Hence each member of Aut $(X)$ is isolated in $\operatorname{Hol}(X, X)$. This implies that $\tilde{\phi}$ is constant, i.e., $\phi(y, \cdot)=\phi\left(y_{0}, \cdot\right)$ on $X$ for every $y \in Y$. This completes the proof.

Proof of Proposition 3. Suppose that $X$ and $Y$ are contained in $C$. Take arbitrarily a complex analytic space $S$ and a holomorphic mapping $\phi: S \times X \times Y \rightarrow X \times Y$ such that $\phi\left(s_{0}, \cdot, \cdot\right) \in$ Aut $(X \times Y)$ for some $s_{0} \in S$. Then we have the holomorphic mapping

$$
\psi=\phi\left(s_{0}, \cdot, \cdot\right)^{-1} \circ \phi: S \times X \times Y \rightarrow X \times Y .
$$

Write $\psi=(\alpha, \beta): S \times X \times Y \rightarrow X \times Y$, where $\alpha$ and $\beta$ are mappings from $S \times X \times Y$ into $X$ and $Y$ respectively. Since $\psi\left(s_{0}, \cdot, \cdot\right)=$ identity on $X \times Y$,

$$
\begin{array}{ll}
\alpha\left(s_{0}, \cdot, y\right)=\text { identity on } X & \text { for any } y \in Y \text { and } \\
\beta\left(s_{0}, x, \cdot\right)=\text { identity on } Y & \text { for any } x \in X .
\end{array}
$$

This implies that $\alpha(s, x, y)=\alpha\left(s_{0}, x, y\right)$ and $\beta(s, x, y)=\beta\left(s_{0}, x, y\right)$ for any $(s, x, y) \in S \times X \times Y$, because $X$ and $Y$ are contained in $C$. Thus we see that $\psi(s, x, y)=\psi\left(s_{0}, x, y\right)=(x, y)$ and hence $\phi(s, x, y)=\phi\left(s_{0}, x, y\right)$ for any $(s, x, y) \in S \times X \times Y$. This means that $X \times Y$ is also contained in $C$.

Conversely, suppose that $X \times Y$ is contained in $C$. Take arbitrarily a complex analytic space $S$ and a holomorphic mapping $\phi: S \times X \rightarrow X$ such that $\phi\left(s_{0}, \cdot\right) \in \operatorname{Aut}(X)$ for some $s_{0} \in S$. Let us define the holomorphic mapping $\psi: S \times X \times Y \rightarrow X \times Y$ by the formula

$$
\psi(s, x, y)=(\phi(s, x), y) \quad \text { for any }(s, x, y) \in S \times X \times Y .
$$

Obviously, $\psi\left(s_{0}, \cdot, \cdot\right) \in \operatorname{Aut}(X \times Y)$. By the assumption, $\psi(s, x, y)=\psi\left(s_{0}, x, y\right)$ for any $(s, x, y) \in S \times X \times Y$. Hence $\phi(s, x)=\phi\left(s_{0}, x\right)$ for any $(s, x) \in S \times$ $X$. This means that $X$ is contained in $C$. Similarly we see that $Y$ is contained in $C$. This completes the proof. 


\section{§3. Lemmas}

In this section we fix the situation as follows:

Let $X, Y, V$ and $W$ be complex analytic spaces. Let $h=(\phi, \psi): X \times$ $V \rightarrow Y \times W$ be a biholomorphic mapping and write $h^{-1}=(\alpha, \beta): Y \times W \rightarrow$ $X \times V$. Take an arbitrary point $x_{0}$ of $X$ and put $Y^{\prime}=\phi\left(x_{0}, V\right):=\left\{\phi\left(x_{0}, v\right)\right.$; $v \in V\}(\subset Y)$ and $W^{\prime}=\psi\left(x_{0}, V\right)(\subset W)$.

Lemma 1. Suppose that $X$ is contained in $C$. Then we have the following:

(1) $Y^{\prime}$ (resp. $W^{\prime}$ ) is a complex analytic subvariety of $Y$ (resp. W) and $h\left(x_{0}, V\right)=Y^{\prime} \times W^{\prime}$ in $Y \times W$; hence $h\left(x_{0}, \cdot\right): V \rightarrow Y^{\prime} \times W^{\prime}$ is biholomorphic.

(2) $\phi\left(x_{0}, \beta(y, w)\right)=y$ for any $(y, w) \in Y^{\prime} \times W^{\prime}$.

(3) $\psi\left(x_{0}, \beta(y, w)\right)=w$ for any $(y, w) \in Y^{\prime} \times W^{\prime}$.

(4) If $Y$ (resp. W) is contained in $C$, then $W^{\prime}$ (resp. $Y^{\prime}$ ) is a direct factor of $W$ (resp. $Y)$.

Proof. (1) Obviously,

$$
h\left(x_{0}, V\right)=\left\{\left(\phi\left(x_{0}, v\right), \psi\left(x_{0}, v\right)\right) ; v \in V\right\} \subset Y^{\prime} \times W^{\prime} .
$$

Consider the holomorphic mapping $\pi:(V \times V) \times X \rightarrow X$ defined as $\pi((v, w)$, $x)=\alpha(\phi(x, v), \psi(x, w))$ for each $((v, w), x) \in(V \times V) \times X$. Evidently, $\pi((v$, $v), \cdot): X \rightarrow X$ is the identity mapping on $X$ for any $v \in V$. Since $X$ is contained in $C$, we see that $\alpha(\phi(x, v), \psi(x, w))=x$ for all $x \in X$ and $v, w \in V$. This means that $h^{-1}\left(Y^{\prime} \times W^{\prime}\right) \subset\left\{x_{0}\right\} \times V$ in $X \times V$. Hence $h\left(x_{0}, V\right)=Y^{\prime}$ $\times W^{\prime}$. Since $h: X \times V \rightarrow Y \times W$ is biholomorphic, $Y^{\prime} \times W^{\prime}$ is a complex analytic subvariety of $Y \times W$ and then $Y^{\prime}$ (resp. $W^{\prime}$ ) is a complex analytic subvariety of $Y$ (resp. $W$ ). Clearly, $h\left(x_{0}, \cdot\right): V \rightarrow Y^{\prime} \times W^{\prime}$ is biholomorphic.

(2) Take any $(y, w) \in Y^{\prime} \times W^{\prime}$. Then $\alpha(y, w)=x_{0}$ and so

$$
\phi\left(x_{0}, \beta(y, w)\right)=\phi(\alpha(y, w), \beta(y, w))=y .
$$

(3) Similarly, for any $(y, w) \in Y^{\prime} \times W^{\prime}$,

$$
\psi\left(x_{0}, \beta(y, w)\right)=w .
$$

(4) Suppose that $Y$ is contained in $C$. Consider the complex analytic space $X^{\prime}=X \times Y^{\prime}$ and the biholomorphic mapping $\gamma$ of $X \times V$ to $X^{\prime} \times$ $W^{\prime}$ defined by the formula

$$
\gamma(x, v)=((x, \bar{\phi}(v)), \bar{\psi}(v)) \in X^{\prime} \times W^{\prime} \quad \text { for each }(x, v) \in X \times V,
$$


where $\bar{\phi}=\phi\left(x_{0}, \cdot\right): V \rightarrow Y^{\prime}$ and $\bar{\psi}=\psi\left(x_{0}, \cdot\right): V \rightarrow W^{\prime}$. Then $h^{\prime}=\gamma \circ h^{-1}: Y$ $\times W \rightarrow X^{\prime} \times W^{\prime}$ is biholomorphic. Write $h^{\prime}=\left(\phi^{\prime}, \psi^{\prime}\right): Y \times W \rightarrow X^{\prime} \times W^{\prime}$. Then $\psi^{\prime}(y, W)=\bar{\psi}(\beta(y, W))=W^{\prime}$ for any $y \in Y^{\prime}$ by (3) above. By (1) applied to $h^{\prime}: Y \times W \rightarrow X^{\prime} \times W^{\prime}, h^{\prime}(y, \cdot): W \rightarrow W^{\prime \prime} \times W^{\prime}\left(\subset X^{\prime} \times W^{\prime}\right)$ is biholomorphic for an arbitrarily fixed point $y$ of $Y^{\prime}$, where $W^{\prime \prime}=\phi^{\prime}(y, W)$. Furthermore

$$
h^{\prime}(y, w)=\left(\left(x_{0}, y\right), w\right) \quad \text { for all } w \in W^{\prime}
$$

because

$$
\phi^{\prime}(y, w)=\left(\alpha(y, w), \phi\left(x_{0}, \beta(y, w)\right)\right)=\left(x_{0}, y\right)
$$

and

$$
\psi^{\prime}(y, w)=\psi\left(x_{0}, \beta(y, w)\right)=w \quad \text { for all } w \in W^{\prime}
$$

by (2), (3) above. This means that $W^{\prime}$ is a direct factor of $W$. If $W$ is contained in $C$, then we see that $Y^{\prime}$ is a direct factor of $Y$ by applying the above argument to the biholomorphic mapping $(\psi, \phi): X \times V \rightarrow W \times Y$. This completes the proof.

Lemma 2. Suppose that $X, Y, V$ and $W$ are contained in $C$ and that $\psi\left(x_{0}, \cdot\right): V \rightarrow W$ is biholomorphic. Then $(1) \psi(x, \cdot)=\psi\left(x_{0}, \cdot\right)$ on $V$ for all $x \in X$ and $(2) \phi(\cdot, v)=\phi(\cdot, w)$ on $X$ for all $v, w \in V$ and, moreover, $\phi(\cdot, v)$ : $X \rightarrow Y(v \in V)$ is biholomorphic.

Proof. Consider the holomorphic mapping $\psi\left(x_{0}, \cdot\right)^{-1} \circ \psi: X \times V \rightarrow V$. Then we see easily that $\psi(x, \cdot)=\psi\left(x_{0}, \cdot\right)$ on $V$ for all $x \in X$, because $V$ is contained in $C$. Hence

$$
h(x, v)=\left(\phi(x, v), \psi\left(x_{0}, v\right)\right) \quad \text { for all }(x, v) \in X \times V .
$$

This implies that $h(\cdot, v): X \rightarrow Y \times\left\{\psi\left(x_{0}, v\right)\right\}(\subset Y \times W)$ is biholomorphic for every $v \in V$, i.e., $\phi(\cdot, v): X \rightarrow Y$ is biholomorphic for every $v \in V$. Since $X$ and $Y$ are contained in $C$, we see easily that $\phi(\cdot, v)=\phi(\cdot, w)$ on $X$ for all $v, w \in V$.

Lemma 3. Suppose that $X$ and $Y$ are contained in $C$ and that $V$ and $W$ are primary. If $\psi\left(x_{0}, \cdot\right): V \rightarrow W$ is non-constant, then $\psi\left(x_{0}, \cdot\right): V \rightarrow W$ is biholomorphic.

Proof. By Lemma 1, $h\left(x_{0}, \cdot\right): V \rightarrow Y^{\prime} \times W^{\prime}$ is biholomorphic, where $Y^{\prime}=\phi\left(x_{0}, V\right)(\subset Y)$ and $W^{\prime}=\psi\left(x_{0}, V\right)(\subset W)$. Note that $\operatorname{dim}_{C} W^{\prime}>0$, 
because $\psi\left(x_{0}, \cdot\right): V \rightarrow W$ is non-constant. Since $V$ is primary and $\operatorname{dim}_{C} W^{\prime}$ $>0$, necessarily $\operatorname{dim}_{c} Y^{\prime}=0$. This means that $\phi\left(x_{0}, \cdot\right): V \rightarrow Y$ is constant and $\psi\left(x_{0}, \cdot\right): V \rightarrow W^{\prime}$ is biholomorphic. Now, by Lemma $1, W^{\prime}$ is a direct factor of $W$. Since $W$ is primary, we conclude that $W^{\prime}=W$. Hence $\psi\left(x_{0}, \cdot\right): V \rightarrow W$ is biholomorphic.

\section{§4. Proofs of Theorem 1 and Corollary 1}

Proof of Theorem 1. Let $X$ be a complex analytic space of the collection $C$ and let $X_{1} \times \cdots \times X_{n}$ be a primary decomposition of $X$. Take another primary decomposition $Y_{1} \times \cdots \times Y_{m}$ of $X$. Since $X_{1} \times \cdots \times X_{n}$ and $Y_{1} \times \cdots \times Y_{m}$ are biholomorphic to $X$, they are contained in $C$. Hence, by Proposition $3, X_{1}, \cdots, X_{n}, Y_{1}, \cdots, Y_{m}, X_{1} \times \cdots \times X_{i}(i=2, \cdots, n)$ and $Y_{1} \times \cdots \times Y_{j}(j=2, \cdots, m)$ are also contained in $C$. Take an arbitrary biholomorphic mapping

$$
f=\left(f_{1}, \cdots, f_{m}\right): X_{1} \times \cdots \times X_{n} \rightarrow Y_{1} \times \cdots \times Y_{m}
$$

Then, after a suitable reordering of $Y_{1}, \cdots, Y_{m}$, we may assume that $f_{m}\left(x_{1}, \cdots, x_{n-1}, \cdot\right): X_{n} \rightarrow Y_{m}$ is non-constant on $X_{n}$ for some $\left(x_{1}, \cdots, x_{n-1}\right)$ of $X_{1} \times \cdots \times X_{n-1}$. By Lemma 3 , we see that $f_{m}\left(x_{1}, \cdots, x_{n-1}, \cdot\right): X_{n} \rightarrow Y_{m}$ is biholomorphic. Furthermore, by Lemma $2, f_{m}\left(x_{1}, \cdots, x_{n-1}, \cdot\right): X_{n} \rightarrow Y_{m}$ is independent of $\left(x_{1}, \cdots, x_{n-1}\right)$ in $X_{1} \times \cdots \times X_{n-1}$ and

$$
\left(f_{1}\left(\cdot, x_{n}\right), \cdots, f_{m-1}\left(\cdot, x_{n}\right)\right): X_{1} \times \cdots \times X_{n-1} \rightarrow Y_{1} \times \cdots \times Y_{m-1}
$$

is a biholomorphic mapping which is independent of $x_{n}$ in $X_{n}$. By these argument, Theorem 1 is easily proved by induction on $n$.

Proof of Corollary 1. Suppose that $V \times X$ is biholomorphic to $V \times Y$ for complex analytic spaces $X, Y$ and $V$ contained in $C$. Let $X_{1} \times \cdots \times$ $X_{\ell}, Y_{1} \times \cdots \times Y_{m}$ and $V_{1} \times \cdots \times V_{n}$ be the primary decompositions of $X$, $Y$ and $V$, respectively. Obviously, $V_{1} \times \cdots \times V_{n} \times X_{1} \times \cdots \times X_{\ell}$ and $V_{1}$ $\times \cdots \times V_{n} \times Y_{1} \times \cdots \times Y_{m}$ are primary decompositions of $V \times X$. Note that $V \times X$ is contained in $C$ by Proposition 3. Hence, by Theorem 1, $\ell=m$ and $\left\{X_{1}, \cdots, X_{\ell}\right\}$ is equal to $\left\{Y_{1}, \cdots, Y_{\ell}\right\}$ as sets of biholomorphic classes of complex analytic spaces. Then $X_{1} \times \cdots \times X_{\ell}$ is biholomorphic to $Y_{1} \times \cdots \times Y_{\ell}$, i.e., $X$ is biholomorphic to $Y$. 


\section{§5. Proof of Theorem 2}

Let $V$ be a hyperbolic complex analytic space and let $X, Y$ be complex analytic spaces such that $V \times X$ is biholomorphic to $V \times Y$. Decompose $X$ and $Y$ by complex analytic spaces $V^{\prime}, V^{\prime \prime}, A$ and $B$ so that the following hold:

(1) $X$ is biholomorphic to $V^{\prime} \times A$ and $Y$ is biholomorphic to $V^{\prime \prime} \times B$.

(2) $V^{\prime}$ and $V^{\prime \prime}$ are hyperbolic.

(3) $A$ and $B$ have no direct factor of positive dimension which is hyperbolic.

Consider the hyperbolic complex analytic spaces $C=V \times V^{\prime}$ and $D=V$ $\times V^{\prime \prime}$. Then we have a biholomorphic mapping $h=(\phi, \psi): C \times A \rightarrow D \times$

$B$. Take any point $x$ of $C$. By Lemma 1, we have the following:

(4) $D^{\prime}=\phi(x, A)$ and $B^{\prime}=\psi(x, A)$ are complex analytic subvarieties of $D$ and $B$, respectively.

(5) $h(x, \cdot): A \rightarrow D^{\prime} \times B^{\prime}$ is biholomorphic.

Since the subvariety $D^{\prime}$ of the hyperbolic complex analytic space $D$ is hyperbolic, $A$ has a direct factor which is hyperbolic. Hence it is necessary that $\operatorname{dim}_{C} D^{\prime}=0$. This means that $\phi(x, \cdot): A \rightarrow D$ is constant for any $x \in C$. Now, write $h^{-1}=(\alpha, \beta)$ on $D \times B$ by holomorphic mappings $\alpha: D$ $\times B \rightarrow C$ and $\beta: D \times B \rightarrow A$. Then, by the similar argument above, $\alpha(z, \cdot): B \rightarrow C$ is constant for any $z \in D$. Thus we have, for arbitrarily fixed points $y_{0} \in A$ and $w_{0} \in B, \alpha\left(\phi\left(x, y_{0}\right), w_{0}\right)=x$ on $C$ and $\phi\left(\alpha\left(z, w_{0}\right), y_{0}\right)=z$ on $D$. This means that $C$ is biholomorphic to $D$. Hence $V^{\prime}$ is biholomorphic to $V^{\prime \prime}$ by Corollary 1. Take any $x_{0} \in C$ and put $\left\{z_{1}\right\}=\phi\left(x_{0}, A\right) \subset$ $D$. Then $\beta\left(z_{1}, \psi\left(x_{0}, y\right)\right)=y$ on $A$ and $\psi\left(x_{0}, \beta\left(z_{1}, w\right)\right)=w$ on $B$, because $x_{0}$ $=\alpha\left(\phi\left(x_{0}, y_{0}\right), w_{0}\right)=\alpha\left(z_{1}, w_{0}\right)=\alpha\left(z_{1}, w\right)$ for any $w \in B$. This means that $A$ is biholomorphic to $B$. Hence $V^{\prime} \times A$ is biholomorphic to $V^{\prime \prime} \times B$, i.e., $X$ is biholomorphic to $Y$.

\section{§6. Proof of Theorem 3}

Decompose $X$ and $Y$ by compact irreducible complex analytic spaces $V^{\prime}, V^{\prime \prime}, X_{1}$ and $Y_{1}$ so that the following hold:

(1) $X$ is biholomorphic to $V^{\prime} \times X_{1}$ and $Y$ is biholomorphic to $V^{\prime \prime} \times Y_{1}$.

(2) $V^{\prime}$ and $V^{\prime \prime}$ are of general type.

(3) $X_{1}$ and $Y_{1}$ have no direct factor of positive dimension which is of general type.

Put $V_{1}=V \times V^{\prime}$ and $V_{2}=V \times V^{\prime \prime}$. Then $V_{1}$ and $V_{2}$ are of general type 
(cf. Ueno [10], p. 69). Since $V \times X$ is biholomorphic to $V \times Y$, we have a biholomorphic mapping $h=(\phi, \psi): V_{1} \times X_{1} \rightarrow V_{2} \times Y_{1}$. Put $V_{v}=\phi\left(v, X_{1}\right)$ $\left(\subset V_{2}\right)$ and $Y_{v}=\psi\left(v, X_{1}\right)\left(\subset Y_{1}\right)$ for each $v \in V_{1}$. By Lemma $1, h(v, \cdot): X_{1}$ $\rightarrow V_{v} \times Y_{v}$ is biholomorphic for every $v \in V_{1}$. Write $h^{-1}=(\alpha, \beta): V_{2} \times Y_{1}$ $\rightarrow V_{1} \times X_{1}$. Now, take any $y_{0} \in Y_{1}$ and put $S=\alpha\left(V_{2}, y_{0}\right)$. Then $S$ is a compact irreducible complex analytic subvariety of $V_{1}$. Consider the holomorphic surjection $\bar{\alpha}=\alpha\left(\cdot, y_{0}\right): V_{2} \rightarrow S$. Then we have the following assertion:

$$
\bar{\alpha}^{-1}(v)=V_{v} \quad \text { for any } v \in S .
$$

Proof of the above assertion. Take any $v^{\prime} \in \bar{\alpha}^{-1}(v)$, where $v \in S$. Then $\alpha\left(v^{\prime}, y_{0}\right)=v$ and hence $\left(v^{\prime}, y_{0}\right) \in h\left(v, X_{1}\right)=V_{v} \times Y_{v}$. Hence $v^{\prime} \in V_{v}$. Conversely assume that $v^{\prime} \in V_{v}$ for some $v \in S$. Since $v=\alpha\left(v^{\prime \prime}, y_{0}\right)$ for some $v^{\prime \prime} \in V_{2}, y_{0}=\psi\left(v, \beta\left(v^{\prime \prime}, y_{0}\right)\right) \in Y_{v}$. Hence $\left(v^{\prime}, y_{0}\right) \in V_{v} \times Y_{v}=h\left(v, X_{1}\right)$. Then $\alpha\left(v^{\prime}, y_{0}\right)=v$, i.e., $v^{\prime} \in \bar{\alpha}^{-1}(v)$. This completes the proof of the assertion.

Now, put $n=\operatorname{Min}\left\{\operatorname{dim}_{c} \bar{\alpha}^{-1}(v) ; v \in S\right\}$. Since $\bar{\alpha}^{-1}(v)=V_{v}=\phi\left(v, X_{1}\right)$ is irreducible for every $v \in S$, we see easily that $U=\left\{v \in S ; \operatorname{dim}_{C} V_{v}=n\right\}$ is open dense in $S$ (cf. Proposition 7 of Holmann [2]). Furthermore, $\operatorname{dim}_{C} V_{2}$ $=n+\operatorname{dim}_{C} S$ by the maximal rank theorem (cf. Narasimhan [12], Chapter VII). On the other hand, by Theorem 6.12 of Ueno [10], there exists an open dense set $U^{\prime}$ of $S$ such that

$$
\kappa\left(V_{2}\right) \leqslant \kappa\left(\bar{\alpha}^{-1}(v)\right)+\operatorname{dim}_{C} S=\kappa\left(V_{v}\right)+\operatorname{dim}_{C} S
$$

for any $v \in U^{\prime}$. Note that $U \cap U^{\prime}$ is also open dense in $S$. Since $V_{2}$ is of general type, i.e., $\kappa\left(V_{2}\right)=\operatorname{dim}_{C} V_{2}$, we obtain

$$
\kappa\left(V_{v}\right)=n=\operatorname{dim}_{C} V_{v}
$$

for any $v \in U \cap U^{\prime}$ because of the general fact that $\kappa\left(V_{v}\right) \leqslant \operatorname{dim}_{C} V_{v}\left(v \in V_{1}\right)$. Hence $V_{v}$ is of general type for any $v \in U \cap U^{\prime}$. Since $X_{1}$ is biholomorphic to $V_{v} \times Y_{v}\left(v \in V_{1}\right)$ and it has no direct factor of positive dimension which is of general type, we obtain $n=0$ necessarily. This means that $\phi(v, \cdot)$ : $X_{1} \rightarrow V_{2}$ is constant for every $v \in U$. In fact $\phi(v, \cdot): X_{1} \rightarrow V_{2}$ is constant for every $v \in S$, because $U$ is dense in $S$. Furthermore, $\operatorname{dim}_{C} V_{2}=\operatorname{dim}_{C} S$ $\leqslant \operatorname{dim}_{C} V_{1}$. By applying the above argument to $h^{-1}: V_{2} \times Y_{1} \rightarrow V_{1} \times X_{1}$, we have $\operatorname{dim}_{C} V_{1} \leqslant \operatorname{dim}_{C} V_{2}$. Hence $\operatorname{dim}_{C} V_{1}=\operatorname{dim}_{C} V_{2}=\operatorname{dim}_{C} S$. By the irreducibility of $V_{1}$, we see that $S=V_{1}$. Consequently $\phi(v, \cdot): X_{1} \rightarrow V_{2}$ is constant for every $v \in V_{1}$. Hence the biholomorphic mapping $h$ has a representation 


$$
h(v, x)=\left(\phi_{1}(v), \psi(v, x)\right)
$$

for each $(v, x) \in V_{1} \times X_{1}$, where $\phi_{1}: V_{1} \rightarrow V_{2}$. Similarly, $h^{-1}$ has a representation

$$
h^{-1}(w, z)=\left(\alpha_{1}(w), \beta(w, z)\right)
$$

for each $(w, z) \in V_{2} \times Y_{1}$, where $\alpha_{1}: V_{2} \rightarrow V_{1}$. We see easily that $V_{1}$ is biholomorphic to $V_{2}$ and $X_{1}$ is biholomorphic to $Y_{1}$. Then, by Corollary $1, V^{\prime}$ is biholomorphic to $V^{\prime \prime}$. Hence $V^{\prime} \times X_{1}$ is biholomorphic to $V^{\prime \prime} \times$ $Y_{1}$, i.e., $X$ is biholomorphic to $Y$.

\section{§7. Example}

In this section complex analytic manifolds are always paracompact and connected. Let $M$ be a complex analytic manifold of complex dimension $n$. Consider the complex vector space $H$ of all holomorphic $n$-forms $f$ on $M$ such that

$$
\int_{M}(\sqrt{-1})^{n^{2}} f \wedge \bar{f}<\infty
$$

Then $H$ is a Hilbert space with the inner product

$$
(f, g)=(\sqrt{-1})^{n^{2}} \int_{M} f \wedge \bar{g} \quad \text { for } f, g \in H .
$$

Further we know that $H$ is a separable Hilbert space. Hence we have the Bergman kernel form $K_{M}$ defined on $M \times M$ (cf. Kobayashi [6], Lichnerowicz [11]). Suppose that

(A.1) Given any point $z$ of $M$, there exists an $f \in H$ such that $f(z) \neq 0$. Then, associated with the Bergman kernel form $K_{M}$ on $M \times M$, we have the positive semidefinite quadratic form $d s_{M}^{2}$ on $M$ which is invariant under the holomorphic automorphism group Aut $(M)$ of $M$ (see [6]). The complex analytic manifold $M$ is called a Kobayashi manifold if the form $d s_{M}^{2}$ is positive definite on $M$; then $d s_{M}^{2}$ is called the Bergman metric on $M$. It has been proved in [6] that, for any complex analytic manifolds $M, N$ of complex dimension $m, n$ respectively,

$$
K_{M \times N}=(-1)^{m n} K_{M} \wedge K_{N} \quad \text { on }(M \times N) \times(M \times N) .
$$

Take any complex analytic manifolds $M$ and $N$ such that $M \times N$ satisfies the condition (A.1) above. Using the Fubini's theorem we see that $M$ and $N$ satisfy the condition (A.1). Then we have 


$$
d s_{M \times N}^{2}=d s_{M}^{2}+d s_{N}^{2} \quad \text { on } M \times N .
$$

Hence $M$ and $N$ are Kobayashi manifolds, if $M \times N$ is a Kobayashi manifold. In fact, the converse is also true (cf. [6]). We obtained that if $M$ is a Kobayashi manifold with a primary decomposition $M_{1} \times \cdots \times M_{m}$ then

$$
\left(M, d s_{M}^{2}\right)=\left(M_{1}, d s_{M_{1}}^{2}\right) \times \cdots \times\left(M_{m}, d s_{M_{m}}^{2}\right) .
$$

By the uniqueness of the (Kähler) de Rham decomposition of Kähler manifolds (cf. Kobayashi and Nomizu [7]), we can conclude that, for any simply connected complete Kobayashi manifold $\left(M, d s_{M}^{2}\right)$, primary decompositions of $M$ are given by the (Kähler) de Rham decompositions of the Kähler manifold $\left(M, d s_{M}^{2}\right)$.

ExAmple 1. Let $D$ be a homogeneous bounded domain of $C^{m}$ (the cartesian product of the complex line $C$ ). Then the Bergman metric of $D$ is complete, and $D$ is simply connected (cf. [3]). Kaneyuki [3] proved that the (Kähler) de Rham decomposition of $D$ is given uniquely by the product of irreducible homogeneous bounded domains $D_{1}, \cdots, D_{n}$ up to isometries. Since $D$ is hyperbolic (cf. Kobayashi [4]), by Theorem 1 we see that $D_{1} \times \cdots \times D_{n}$ is the unique primary decomposition of $D$. Furthermore, if each $D_{i}$ is not biholomorphic to $D_{j}(i \neq j=1, \cdots, n)$, then $\operatorname{Aut}(D)=\operatorname{Aut}\left(D_{1}\right) \times \cdots \times \operatorname{Aut}\left(D_{n}\right)$ by Corollary 2 .

\section{REFERENCES}

[1] J. Brun, On the cancellation problem for compact complex-analytic manifolds, Proceedings in Symposia of Pure Mathematics, 30 (1977), 247-249.

[2] H. Holmann, Local properties of holomorphic mappings, Proc. Conf. on Complex Analysis, Minneapolis (1964), 94-109.

[ 3 ] S. Kaneyuki, On the automorphism group of homogeneous bounded domains, J. Fac. Sci. Univ. Tokyo, Sect. I, 14 (1967), 89-130.

[4] S. Kobayashi, Hyperbolic manifolds and holomorphic mappings, Marcel Dekker, 1970.

[ 5 ] - Intrinsic distances, measures and geometric function theory, Bull. Amer. Math. Soc., 83 (1976), 357-416.

[6] - Geometry of bounded domains, Trans. Amer. Math. Soc., 92 (1959), 267-290.

[ 7 ] S. Kobayashi and K. Nomizu, Foundations of differential geometry, Vol. II, Interscience Publishers, 1969.

[ 8 ] K. Peters, Starrheitssätze für Produkte normierte Vektorräume endlicher Dimension und für Produkte hyperbolischer komplexer Räume, Math. Ann., 208 (1974), 343-354.

[ 9 ] H. L. Royden, Holomorphic fiber bundles with hyperbolic fiber, Proc. Amer. Math. Soc., 43 (1974), 311-312.

[10] K. Ueno, Classification theory of algebraic varieties and compact complex spaces, 
Springer Lecture Notes in Math., 439 (1975).

[11] A. Lichnerowicz, Variétés complexes et tenseur de Bergman, Ann. Inst. Fourier (Grenoble), 15 (1965), 345-407.

[12] R. Narasimhan, Introduction to the theory of analytic spaces, Springer Lecture Notes in Math., 25 (1966).

Department of Mathematics

Aichi University of Education

Kariya-shi, 448 Japan 